\title{
Biomarkers in Parkinson's disease - An effective tool for diagnosis of PD
}

\author{
Anitha Nandagopa $\left.\right|^{1 *}$, Syeda Nishath Sultana ${ }^{2}$, Anupama Koneru ${ }^{3}$ \\ 1 Professor and HOD, Dept. of Pharmacology, Sultan-ul-Uloom College of Pharmacy, Road no.3, Banjara Hills \\ Hyderabad - 500034, Telangana, India. \\ 2 M.Pharm, II-Year, Dept. of Pharmacology, Sultan-ul-Uloom College of Pharmacy, Road no.3, Banjara Hills, \\ Hyderabad - 500034, Telangana, India. \\ 3 Principal and Professor, Sultan-ul-Uloom College of Pharmacy, Road no.3, Banjara Hills, \\ Hyderabad - 500034, Telangana, India.
}

\begin{abstract}
Parkinson's disease (PD) ranks the second position of neurodegenerative disorder mainly arises due to depleted levels of neurotransmitter dopamine in substantia nigra. PD is characterized by decreased levels of dopamine due to dopaminergic cell death and manifested by tremor, rigidity, bradykinesia and postural abnormalities. Considerable latency is observed in dopaminergic cell death and appearance of clinical symptoms in PD, so it is necessary to diagnose the disease in early stages for better treatment. Establishment of reliable biomarkers which indicates the progression of the disease and will aid in better clinical management of PD patients. Biomarkers when coupled with system biology tools help in discovering transcriptomic, proteomic, genomic and metabolic molecules and other signaling pathways responsible for differential diagnosis of parkinsonism disorder and serves as tool in monitoring disease progression. Identifying the subjects at earlier stage of disease would pave the way to novel drug therapies to decrease the progression of disease.
\end{abstract}

Keywords: Parkinson's disease, LRRK2, DJ-1biomarker, neuroimaging biomarkers, reflex tears, $\alpha$-synuclein

\section{INTRODUCTION}

Parkinson's disease (PD) is a complex syndrome with different clinical subtypes characterized by a large motor and non-motor features which intern effect the

\section{*Correspondence:}

Dr. Anitha Nandagopal, NProfessor and HOD, Dept. of Pharmacology, NSultan-ul-Uloom College of Pharmacy, Road no.3, Banjara Hills, Hyderabad - 500034, Telangana, India.

Email: anirajan_76@yahoo.co.in Mobile: 09959971590

ORCIDs

Anitha Nandagopal: 0000-0002-4294-9412

Syeda Nishath Sultana: 0000-0001-9601-5220

Anupama Koneru: 0000-0002-3497-7114

(Received 29 February 2020, accepted 26 July 2020) 
function and quality of life of affected individuals. Pathological evidences suggest that the pathological process of PD begins much earlier prior to the appearance of typical motor symptoms. About $70-80 \%$ of striatal dopamine and one third of substantia nigra neurons and striatal dopaminergic neurons have already been lost before the diagnosis is made. ${ }^{1,2}$

Pathology of PD is complicated. Recent studies have reported the evaluation of brain samples of PD patients showing the presence of Lewy bodies (LB) which is the hallmark of PD to trace the damaged neurons throughout the nervous system. Braak et al conducted a study and reported that there are six neuropathological stages of $\mathrm{PD}^{3}$, which are shown in Fig 1.

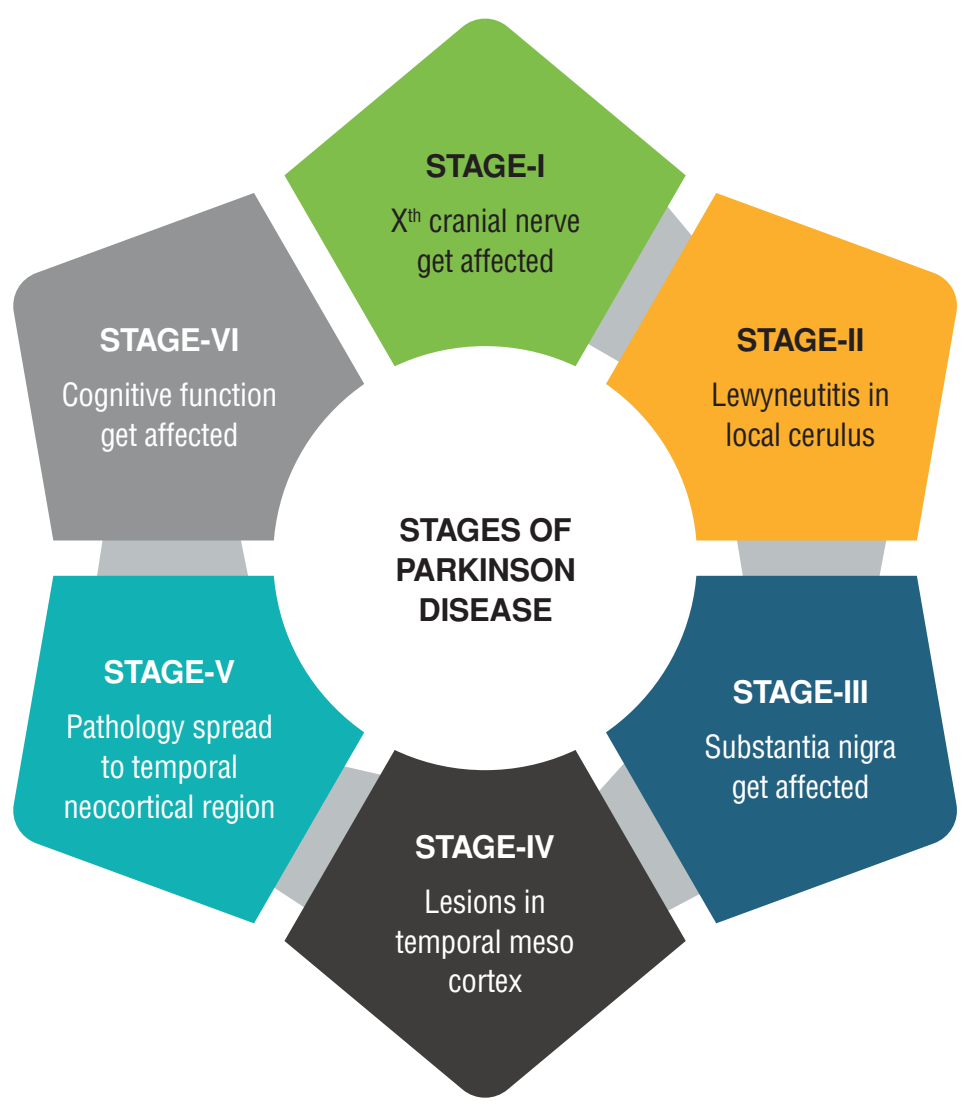

Figure 1. Neuropathological stages of Parkinson disease 
STAGE-I: In this stage beginning of pathology is traced in lower medulla oblongata in the dorsal motor nucleus of the tenth cranial nerve and the olfactory structures specifically in anterior part.

STAGE-II: Lewy neuritis is observed in locus coeruleus, exacerbation of lesions in dorsal motor nucleus.

STAGE-III: Damage to the substantia nigra is observed.

STAGE-IV: Presence of lesions in the temporal mesocortex.

STAGE-V: Pathology is seen in the adjoining temporal neocortical fields.

STAGE-VI: Cognitive status correlates with the neuropathological stage.

According to National Institute of Health (NIH), a biomarker is a tool which assists in detection and diagnosis of disease and indicates the pharmacological response to a therapeutic intervention. ${ }^{4}$ Development of reliable and sensitive biomarkers will help in detection of individual at risk before the progression of classical symptoms in a stage where neuroprotective therapies have higher efficacy. Establishment of a reliable biomarker will help in discriminating PD from other neurodegenerative disorders. Biomarkers are the tools which assist the physicians in developing pathogenesis-targeted therapeutics of PD. 5

\section{Biomarkers in PD}

Parkinsonism is manifested by motor features including bradykinesia, tremors, rigidity and postural abnormalities along with the non-motor features. Myriad of biomarkers are used at different stages of PD. Different phases of PD are shown in Figure 2.

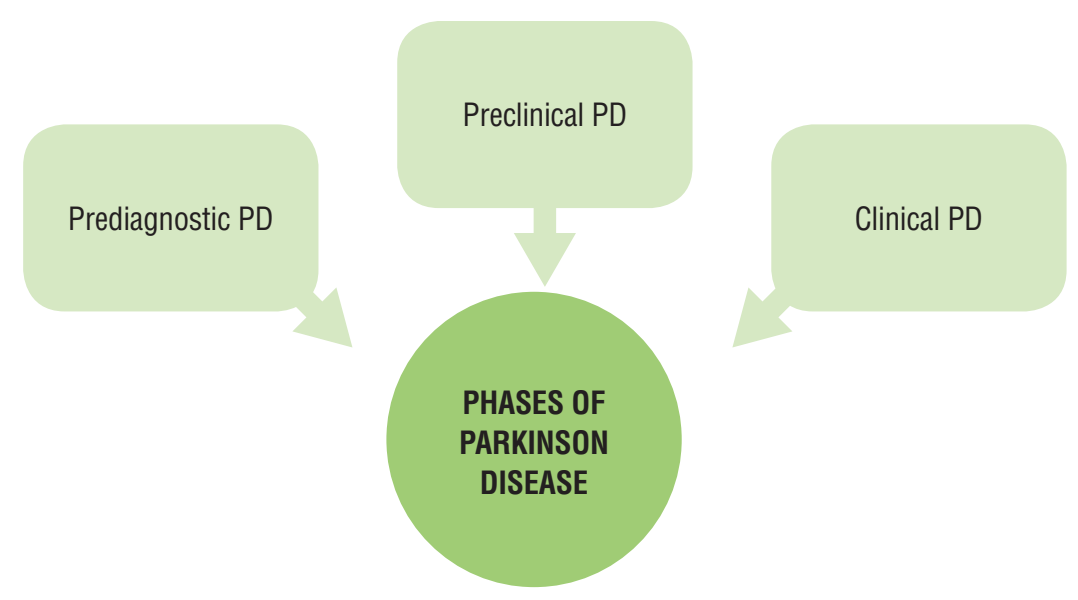

Figure 2. Different phases of Parkinson's disease 


\section{Prediagnostic phase of PD}

In established PD, patients experience non-motor symptoms which are as troublesome as motor features, and treatment is often difficult. Non-motor symptoms are experienced early and there is substantial evidence which suggests that they also aid in early diagnosis of the disease several years prior to the appearance of clinical symptoms. A number of studies have demonstrated the association of PD with earlier diagnosis such as depression, anxiety, constipation and erectile dysfunction. $6,7,8$

\section{Biomarkers in prediagnostic stage}

The clinical diagnosis of PD requires multiple motor features to be established, and while subtle motor signs may be present, a clinical diagnosis of PD is not possible until motor symptoms become more definite. ${ }^{9}$ Non-motor features potentially serves as biomarkers in early diagnosis of PD. Environmental factors also contribute to the occurrence of PD. Exposure to pesticides in farmers and people living in rural areas are associated with increased risk of PD. Rotenone and paraquat which are chemically relative to MPTP (1-methyl-4-phenyl1,2,3,6-tetrahydropyridine specifically increase the risk of PD. ${ }^{10}$ Mendelian randomisation (MR) study is a powerful technique which correlates between the risk factors and progression of the disease. This technique was used by Simon and colleagues, they used polymorphism of single nucleotide in SLC2A9 gene to estimate the genetic variability of serum urate levels. Outcome of this study suggested elevated levels of urate in serum which delayed progression of the disease. Findings of this MR study emerged as novel therapeutic strategy to treat the disease progression by modulating therapeutic urate levels. ${ }^{11}$

\section{Biomarkers in preclinical phase of PD}

Preclinical phase is that stage of PD which precedes the diagnostic phase of PD which is devoid of motor symptoms of the disorder characterised by subtle non-motor features of the disease which can be detected by physiological examination. Various biomarkers are available for determining preclinical stage which may develop PD in future. ${ }^{12}$ Biomarkers in preclinical phase of PD are represented in Figure 3. 


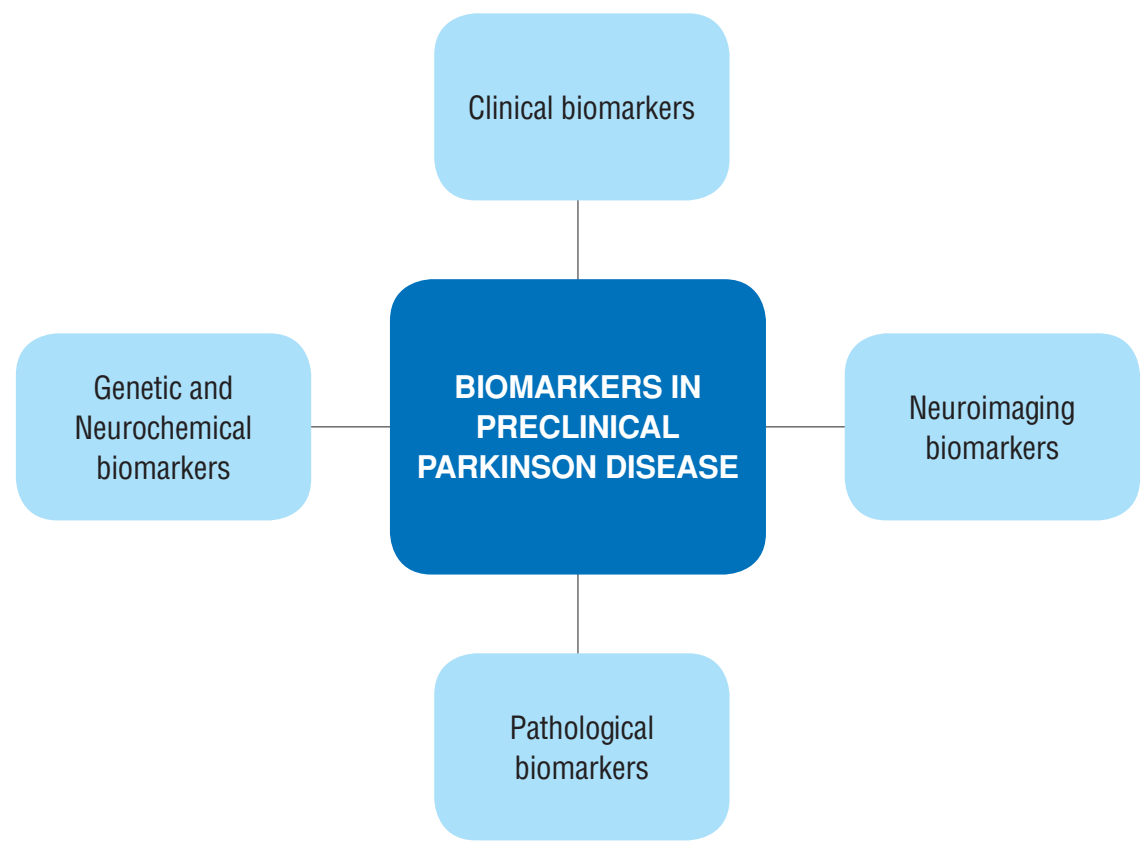

Figure 3. Biomarkers in preclinical stage of Parkinson's disease

\section{Clinical biomarkers of preclinical pd}

Evidences suggest that prior to the appearance of motor features of PD various premonitory sign and symptoms are seen ${ }^{13}, 14$ which are as follows,

\section{Sleep Disturbances}

Patients with PD have disturbed sleep pattern characterised by rapid behavioural disorder (RBD) which is seen much prior to the onset of motor symptoms. ${ }^{15}{ }^{16}$ In a long-term clinical study conducted with 93 subjects with RBD, 14 patients developed Parkinsonism with high degree of cognitive impairment. ${ }^{17}$

\section{Essential tremors}

Neuropathological studies and epidemiological studies have suggested that patients with essential tremors have co-existent PD. ${ }^{18}$ Cohort study conducted on 3813 geriatric patients in central Spain reported that patients with Essential tremors are 4 times more prevalent to develop PD than controls. ${ }^{19}$

\section{Olfactory Dysfunction}

Population based prospective studies revealed the association between olfactory loss and future development of PD. ${ }^{20}$ About 90\% of the patients suffer- 
ing from PD exhibits anosmia. ${ }^{21,22}$ Odour separation measures indicate the severity of disease and further studies support that olfactory dysfunction is the early sign of idiopathic PD. Neuroimaging and neurochemical marker studies revealed that olfactory loss in PD can be seen 2 to 7 years prior to the clinical development of PD. ${ }^{23}$

\section{Constipation}

Dysautonomia is generally traced at the advanced stages of PD. According to epidemiological studies conducted among the various symptoms of autonomic dysfunction constipation appears earliest and much prior to motor symptoms. ${ }^{24}$

\section{Pathological biomarkers in preclinical pd}

Degeneration of dopaminergic neurons in substantia nigra pars compacta, associated with proteinaceous inclusions within the cytoplasm known as Lewy Bodies (LB) is the crucial pathological hallmark of PD.

Braak hypothesis however suggested that neurodegeneration of non-dopaminergic neurons in the caudal regions of brainstem particularly in dorsal motor nucleus of vagus and olfactory regions precedes the dopaminergic degeneration. Braak hypothesis states that $\alpha$-synuclein pathological changes in PD begin in the lower brainstem and progress in caudal-rostral pattern. This promotes the notion that non-motor features reflecting this prenigral involvement can precede the classic motor features of PD.

Based on the study conducted by implanting embryonic dopaminergic cell in the striatum of patients with PD have developed pathological features including formation of LB has led to the hypothesis that $\alpha$-synuclein can be secreted into extracellular space of brain and spread PD in a prion like fashion. ${ }^{25}$

Olfactory features

According to Hawkes et al synucleinopathy in PD starts in olfactory bulb and enteric plexuses this paved the way to dual-hit hypothesis according to this an unknown neurotropic pathogen initiates the pathogenesis underlying sporadic PD in anterograde and retrograde fashion. ${ }^{26}$

Skin biopsy

By performing punch skin biopsy using immune staining and panneuronal marker for protein gene product, has shown the involvement of peripheral autonomic nervous system in early stages of PD. Presence of $\alpha$-synuclein deposits in dermis of PD patients concludes it as variant synucleinopathy. ${ }^{27,28}$ 


\section{NEUROIMAGING BIOMARKERS IN PRECLINICAL PD}

Presymptomatic stages of $\mathrm{PD}$ can be detected by various imaging techniques. ${ }^{29}$ Brain abnormalities in PD patients using various techniques such as dopamine transporter-single photon emission computed tomography (DAT-SPECT), positron emission tomography (PET), transcranial B-mode sonography (TCS), diffusion weighted imaging (DWI), magnetic resonance imaging (MRI), sonography.

\section{Positron Emission Tomography}

PET scan involves emission of positrons which are positively charged. DATSPECT at the straiatal regions of substantia nigra can be assessed employing radiolabeled dopamine analogs such as ${ }^{18} \mathrm{~F},{ }^{11} \mathrm{C},{ }^{18} \mathrm{~F}$-dopamine ${ }^{30},{ }^{18} \mathrm{~F}$-FE-PE2I, ${ }^{18} \mathrm{~F}$-LBT999 ${ }^{31}$, ${ }^{11} \mathrm{C}$-methylphenidate, ${ }^{11} \mathrm{C},{ }^{18} \mathrm{~F}$ radiolabelled dihydrotetrabenazine (DTBZ) are the radioligands used for quantification of vesicular monoamine transporter type 2 (VMAT2).

Both DAT and VMAT2 ligands trace the early signs of dopaminergic damage. ${ }^{32}$ DAT ligands estimate the uptake and decarboxylation of levodopa coupled with the amount of radiolabeled dopamine stored in vesicles. Reduced DAT binding is the earliest indication of dopaminergic dysfunction in individuals and serves as potential biomarker of preclinical PD. ${ }^{33}$ Another radiolabelled ${ }^{11} \mathrm{C}$-dihydrotetrabenazine detects nigrostriatal deficits in preclinical PD indicating the severity of PD.

\section{DAT SPECT}

Single photon emission computed tomography (SPECT) imaging technique aids in visualising the dopamine transporter (DAT) levels in the brain. Studies revealed reduced binding of DAT in patients with PD which further indicates the severity and asymmetry of motor clinical scores. ${ }^{34}$ Binding of N-fluoropropyl-2b-carbomethoxy-3b-4-[123I] iodophenyl-nortropane ([123I]-FP-CIT) to the striatum is helpful in detecting the severity even at preclinical stage in subjects with de novo PD, thus any abnormality in imaging serves as fruitful biomarker of early PD. 35

\section{Trans Cranial B-Mode Sonography (TCS)}

Most reliable method used to measure the velocity of blood flowing through brain vessels by measuring the echoes and frequency of ultrasound waves. When patients with PD are subjected to TCS increased echogenicity of substantia nigra is traced in the brains of PD patients. According to Skoloudik et al., higher echogenicity is mainly due to elevated levels of iron, gliosis due to alteration or malfunction of blood brain barrier. ${ }^{36}$ 


\section{Magnetic Resonance Imaging (MRI)}

MRI is a form of DWI (Diffused Weighted Imaging) which is based on diffusion principle. It measures the amount of water diffused through the tissue thereby determines the structural defects. Increased diffusivity of water indicates the necrosis of cells or reduced volume of the tissue region. This technique also distinguishes PD from multiple system atrophy (MSA). Patients with MSA exhibits more diffusivity of water in the middle cerebella peduncles compared to PD patients. ${ }^{37}$ High resolution 3-Tesla MRI can also trace the diminished volume of caudate and putamen in PD patients. ${ }^{38}$

\section{Genetic Biomarkers in preclinical PD}

Alpha-Synuclein - Levels of $\alpha$-synuclein in PD patients's CSF have been found to be decreased which is due to syucleinopathy. ${ }^{39}$ Thus $\alpha$-synuclein is employed as a potential biomarker in clinical diagnosis of PD. ${ }^{40}$

LRRK2 (OMIM*609007) - Mutations in LRKK2 is responsible for 10-15\% of familial cases with autosomal dominant PD, ${ }^{41} 30-40 \%$ of familial cases are mainly due to mutations in LRRK2 such as G2019S which was evolved as a potential screening tool in Ashkenazi Jews and North African Arabs. ${ }^{42,43}$

Multi functional protein DJ-1(OMIM*602533) - Decreased levels of this protein is responsible for familial, Autosomal recessive PD. ${ }^{44}$

Parkin gene (OMIM*601828) - Mutation of parkin gene is major cause of several tumors and autosomal recessive PD. ${ }^{45}$

Transcription factor NURR1 (NR4A2) (OMIM*601828) - It is a member of nuclear receptor family essential for the development, survival, functional maintenance of dopaminergic neurons in midbrain. ${ }^{46} \mathrm{~A}$ study conducted on 278 subjects suffering from PD, 166 healthy subjects and 256 controls with neurological disease and the results revealed that there is decreased expression of NURR1 gene in PD patients. ${ }^{47}$

Cocaperone $\mathbf{s t}_{\mathbf{1}_{3}}$ (omim*606796) - It stabilizes Heat shock protein-70 (HSP-70) which has the ability to modify a-synuclein misfolding and toxicity. Levels of ST13 m-RNA was significantly reduced in PD patients. ${ }^{8}$

\section{Biomarkers in clinical phase of pd}

Clinical phase of PD is associated with motor symptoms such as bradykinesia, rigidity and resting tremors. Detection of motor signs and symptoms serves as potential markers in progression of disease at different stages. NIH sponsored a clinical trial study named DATATOP for determining motor scores to identify 
disease progression. Several non-motor features precede the classical motor features. Such as olfactory dysfunction, REM sleep disorder, bowel dysfunction and depression serve as markers of disease. ${ }^{49}$

\section{Neurochemical biomarkers in parkinson's disease}

\section{Orexin}

Lateral and posterior neurons of hypothalamic region possess a neuropeptide hormone named orexin also known as hypocretin. It has a key role in regulating sleep-wake cycle, ${ }^{50}$ cardiovascular response, hypertension ${ }^{51}$ and heart rate. Narcolepsy seen in PD patients is mainly due to degeneration of hypocretin neurons in hypothalamus. Severity of disease is directly related to the concentration of orexin. ${ }^{22}$ Orexin levels are decreased in PD patients than healthy subjects.

Glial fibrillary acidic protein (GFAP) in CSF is expressed by astrocytes. Increased GFAP levels mainly responsible for decreased levels of orexin, thus GFAP is considered as potential biomarker. ${ }^{53}$

\section{8-Hydroxy-2'-Deoxyguanosine (8-OHdG)}

It is a reactive oxygen species (ROS) of 8-hydroxyguanine responsible for DNA lesions. ${ }^{54}$ Increased serum levels of $8-\mathrm{OHdG}$ are observed in PD. Hence, 8-OHdG serves as potential biomarker in PD. ${ }^{5}$

\section{Dopamine transporter (DAT)}

Increased dopamine levels are toxic to the neurons by undergoing oxidation to form reactive quinones. In order to maintain the levels of dopamine, dopamine transporters such as vesicular monoamine transporter 2 (VMAT 2) store excess dopamine in vesicles. Any change in dopamine transporter levels is an indicator of PD. ${ }^{56}$

\section{Dopamine metabolites}

Decreased levels of 3, 4-dihydroxy phenyl acetic acid (DOPAL) and DOPA in CSF are helpful in diagnosing preclinical PD. DOPAL is responsible for oligomerization and aggregation of $\alpha$-synuclein in PD. ${ }^{57}$

\section{Dopamine Receptor}

Among the five different types of dopamine receptors (D1-D5), D3 type of receptor is significantly reduced due to reduced dopamine signals [55]. Therefore, $\mathrm{D}_{3} \mathrm{R}$ is used as a potential biomarker for PD. ${ }^{8}$

\section{Apolipoprotein A1 (APO A1)}

Liver and small intestine are the major sources of APO A1 which is an essential constituent of high-density lipoprotein (HDL). APOA1 along with APOE are in- 
volved in lipid transportation in the brain. ${ }^{59} \mathrm{CSF}$ of PD patients show reduced levels of APOA1and tetranectin have been observed, thus act as a potential biomarker for PD. ${ }^{60,61}$

\section{RNA-Based PD Biomarkers}

A novel diagnostic approach is developed to identify PD in early stages. MiRNA's are non-coding which consists of 21-24 nucleotides emerged as potential biomarkers of PD because of their presence in CSF as well as in exosome. A study conducted by Dos Santos et al analyzed expression of miRNA profile using NGS (next generation sequencing) in CSF of PD patients and identified miRNA based biomarker pannel of Let-7f-5p,miR-125a-5p,miR-151a-3p,miR27-a-3p and miR-423-5p five best ranking variables. ${ }^{62}$

\section{Peripheral Proteasome and Caspase Activity}

PD is associated with accumulation of intracellular inclusions called Lewy's bodies (LB). Mutations in proteasomes lead to their dysfunction and potentiates accumulation of aggregated $\alpha$-synuclein. ${ }^{63}$ Increased ROS in case of mitochondrial deficiency increases oxidation of $\alpha$-synuclein leading increased ATPindependent proteosomal activity and greater $\alpha$-synuclein oligomerization. Any changes in ATP levels inhibit 26s proteasome. ${ }^{64}$ Severity of the disease is associated with decreased proteosome 20 activity and increased caspase 3 activity. Raised levels of caspase 3 initiate apoptosis thereby decrease proteasome activity. Therefore, proteasome and caspase are used as potential biomarkers. ${ }^{65}$

\section{Novel Biomarkers in PD}

Proteomics, metabolomics and transcriptomics are novel tools capable of tracing any changes in protein, metabolites, RNA profiles and body fluids to distinguish healthy subjects and diseased subjects. ${ }^{66}$

\section{CSF fluid metabolite profile}

Pathological changes in brain are directly related to composition abnormalities of CSF. PD is associated with neurodegenerative changes of dopaminergic neurons in nigrostriatal pathway. Levels of dopamine and its metabolites determine the progression and severity of the disease and are considered to be potential biomarkers.

CSF fluid is analyzed by various analytical techniques, one of the analytical techniques used for analyzing CSF is LCECA (LC-electrochemistry array). Reduced levels of homovanillic acid (HVA), dihydoxyphenylaceticacid (DOPAC), L-Dopa and dihydroxyphenylglycol have been reported in PD patients. ${ }^{67}$ 
Another analytical technique used is gas chromatography-time of flight/ mass spectrometry (GC-TOF/MS), various metabolites such as tryptophan, creatinine, 3-hydroxyisobutyricacid; glucose mannose levels are reduced in PD patients. ${ }^{68,69}$

\section{Blood profile}

Screening of blood of healthy and diseased subjects suffering from PD led to the discovery of novel biomarkers for diagnosis of the disease. Dysregulation of kynurenine pathway is seen in PD patients. ${ }^{70,71,72}$ The changes in kynurenine metabolites in PD serves as biomarker and also pave the way to novel therapeutic strategy for treating PD by giving kynurenic acid supplement or by reducing quinolinic acid using kynurenine 3-monooxygenase inhibitors. Urate levels in blood have significant importance in determining the progression and severity of the disease. Urate is an important antioxidant present endogenously. ${ }^{73}$ Attributing to its antioxidant property increased urate levels help in fighting against oxidative stress in PD patients. High urate levels indicate low risk and slow progression of the disease. ${ }^{74,75}$ These findings led to the longitudinal study of a nutritional supplement inosine which increases urate levels so used in treating PD.

Sometimes misdiagnosis of PD due to presence of co-existing neurodegenerative diseases such as progressive supra-nuclear palsy (PAP), primary progressive multiple sclerosis (PPMS), multiple system atrophy (MSA) may misled the diagnosis and treatment.

NMR (nuclear magnetic resonance) and LC-MS (liquid chromatographymass spectrometry) study reports revealed that branched chain amino acids (BCAA'S) levels are high in patients with PD, PAP, MSA compared to healthy subjects. ${ }^{76} \mathrm{~N}$-8-acetyl sperm dine is effective in determining disease onset and progression of the disease. In addition to the biomarker activity it also plays significant role in delaying or slowing down the progression of the disease. ${ }^{77}$

\section{Urine analysis}

Biopyrrin is the oxidative product of bilirubin. Biopyrrin exacerbates the oxidative stress associated with PD and identified as novel biomarker for sporadic PD. ${ }^{78}$ Urine being a rich source of metabolites is screened by GC-MS and LCMS techniques. Results revealed that dysregulation of steroidogenesis, tryptophan, and phenylalanine pathways are related to the progress of the disease.

\section{Fecal examination}

Fecal short chain fatty acids (SCFAs) promote GI motility and regulate functions of ENS (enteric nervous system). GC-based analysis reported the decreased levels of SCFAs are responsible for development of gastrointestinal motility disorders in PD patients compared to controls. ${ }^{79}, 80$ 


\section{Other Naive Biomarkers}

\section{Reflex tears}

Reflex tears emerged as novel biomarker for diagnosis of PD. Reflex tearing involves the sensory and afferent nerve fibers which are damaged in PD. A study conducted involving 84 PD patients and 84 healthy controls. Oligomeric $\alpha$-synuclein ( $\alpha$-syn oligo), total $\alpha$-synuclein, lactoferrin and matrix metalloproteinase 9 levels are raised in tears of PD patients compared to healthy controls. Flow rate is significantly reduced in PD patients. ${ }^{81,82}$

\section{Saliva $\alpha$-synuclein}

A study conducted at Al-Mustansiriya University engaged 20 PD patients and 20 healthy subjects. Each subject is tested for salivary flow rate, salivary protein and $\alpha$-synuclein using ELISA (Enzyme linked immune sorbent assay). Unstipulated saliva collected from each subject and it is screened through ELISA. Saliva a-synuclein levels is less in PD patients compared to healthy subjects. Therefore, saliva $\alpha$-synuclein can be used as a potential biomarker in PD. ${ }^{83}$

\section{Interleukins (IL-6, IL-10, IL-12)}

High plasma concentration of proinflammatory agents like IL-6, IL-10 and IL12 increases the risk of PD and thereby causing immunological disturbances in PD patients. ${ }^{84,85}$

\section{Low density lipoprotein}

A study involving 236 PD patients and healthy controls reported that reduced LDL levels increase the risk of PD. This further promotes the novel therapeutic strategy of decreasing the risk of PD by increasing cholesterol levels. ${ }^{86,87}$

\section{Hair color}

In PD patients the dark color of hair found to be decreased. Red hair colored and blond subjects are at high risk of PD and have a role of pigmentation in pathogenesis of PD. ${ }^{88}$

All the above-mentioned naive biomarkers are interesting and worth but need to be validated to consider as potent biomarkers. Figure 4 represents the various biomarkers of PD. 


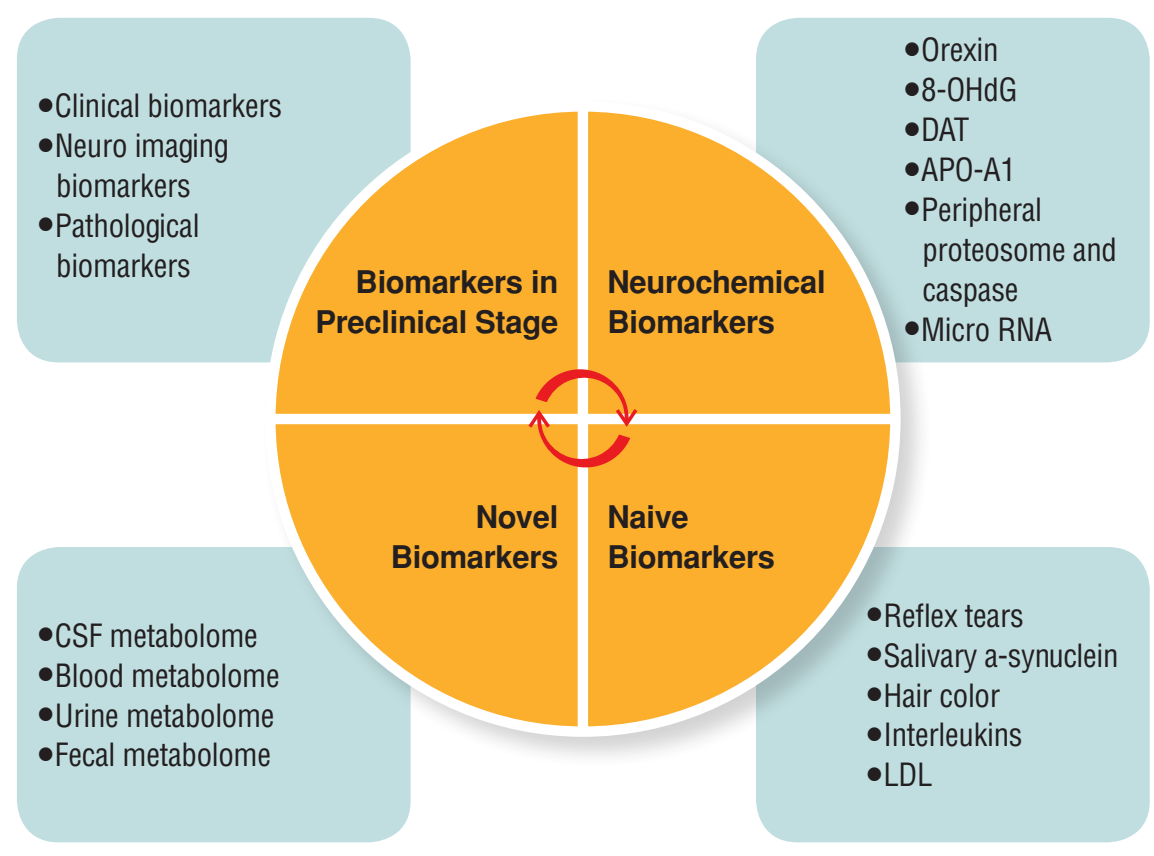

Figure 4. Biomarkers in Parkinson's disease

\section{RESULTS and CONCLUSION}

PD is the second most common neurodegenerative disorder affecting more than 10 million individuals worldwide. Because of considerable time lapse between neuronal damage and appearance of clinical symptoms, it is essential to discover and develop the biomarkers and diagnostic tools for prognosis of the disease and developing novel strategies in treatment. Quality of life improvement is the most urgent and unmet need in the field of science. Biomarkers are the unique tools which ease the process of diagnosis of the disease at early stages to identify at-risk individuals. Imaging techniques such as DAT-SPECT, MRI and PET are available but because of high cost and nonspecific target these techniques are not validated at preclinical stages. Many neurochemicals, non-motor features such as anosmia, olfactory dysfunction, constipation emerged as successful biomarkers for future diagnosis of PD pathology. Newly introduced biomarkers such as reflex tears, salivary levels of $\alpha$-synuclein gathered considerable attention but they need to be validated to use them as potential biomarkers.

Single parameter is unlikely to become a fruitful biomarker. Intensive examination is to be done to introduce potent biomarkers which further potentiate the development of therapeutic strategies to increase the quality of life of patients 
by prognosis of the disease. Studies which allow the assessment of combined data from various fields such as clinical, laboratory, imaging, and genetics must be preferred. Current review is presented with myriad of biomarkers and their ability to develop the novel therapeutic strategies in treating PD but these findings are just the "tip of the iceberg". In order to get access to the wider picture of PD etiology in-depth biological investigation has to be done. Discovery of miRNAs is a breakthrough in field which is a growing hope that screening these miRNAs greatly contribute in diagnosing the disease at early stages. Parkinson's Progression Markers Initiative (PPMI) is conducting an observational clinical study aimed to evaluate the data obtained through cohort studies employing patients with PD along with healthy subjects utilizing advanced imaging, sampling coupled with other laboratory biomarkers using body fluids to identify biomarkers of Parkinson disease progression. With the application of novel techniques such as proteomics, metabolomics, genomics and transcriptomics, it is likely to develop biomarkers which aid in detecting asymptomatic individuals with early disease so that they get benefit from early therapeutic intervention.

\section{ACKNOWLEDGEMENT}

The authors are grateful to Sultan-ul-Uloom Educational Society for providing us the facility to carry out this work.

\section{CONFLICTS OF INTERESTS}

There are no conflicts of interest among the authors. 


\section{REFERENCES}

1. Bohnen, N.I.; Albin, R.L.; Koeppe, R.A.; Wernette, K.A.; Kilbourn, M.R.; Minoshima, S.; Frey, K.A. (2006) Positron emission tomography of mono-aminergic vesicular binding in aging and Parkinson disease. J Cereb Blood Flow Metab. 26(9):1198-1212.

2. Graford, S.; Very, M.; Bonnet, A.M.; Beinis, J.Y.; Gallinari, C.; Meaume, S.; Piette, F.; Hauw, J.J.; Duyckaerts, C. (2006). Motor score of the Unified Parkinson Disease Rating Scale as a good predictor of Lowy body-associated neuronal loss in the substantial Ingra. Arch Neurol. 63(4):584-588.

3. Braak, H.; Del Tredici, K.; Rüb U.; De Vos R.A.; Jansen Steur, E.N.; Braak, E. (2003). Staging of brain pathology related to sporadic Parkinson's disease. Neurobiol Aging. 24(2):197-211.

4. Hiker, R.; Schweitzer, K.; Co burger, S.; Ghaemi, M.; Weisenbach, S.; Jacobs, A.H.; Rudolf, J.; Herholz, K.; Heiss, W.D. (2005). Nonlinear progression of Parkinson disease as determined by serial positron emission topographic imaging of striatal fluorodopa $\mathrm{F} 18$ activity. Arch Neurol. 62(3):378-382.

5. Chaudhuri, K.R.; Schapira, A.H. (2009). Non-motor symptoms of Parkinson's disease: dopaminergic pathophysiology and treatment. Lancet Neurol. 8:464-474.

6. Noyce, A.J.; Bestwick, J.P.; Silveira-Moriyama, L.; Silveira-Moriyama, L.; Hawkes, C.H.; Giovannoni, G.; Lees, A.J.; Schrag, A. (2012). Meta-analysis of early nonmotor features and risk factors for Parkinson disease. Ann Neurol. 72:893-901.

7. Schrag, A.; Horsfall, L.; Walters, K.; Noyce, A.; Petersen, I. (2015). Prediagnostic presentations of Parkinson's disease in primary care: a case-control study. Lancet Neurol. 14:57-64.

8. Adams-Carr, K.L.; Bestwick, J.P.; Shribman, S.; Lees A.; Schrag, A.; Noyce, A.J. (2015). Constipation preceding Parkinson's disease: a systematic review and meta-analysis. J Neurol Neurosurg Psychiatry. 87(7):710-716.

9. Gibb, W.R.; Lees, A.J. (1988). The relevance of the Lewy body to the pathogenesis of idiopathic Parkinson's disease. J Neurol Neurosurg Psychiatry. 51:745-752.

10. Nicholson, J.K.; Connelly, J.; Lindon, J.C.; Holmes, E. (2002). Metabolomics: a platform for Studying drug toxicity and gene function. Nat Rv Drug Discov. 1:153-161.

11. Grimes, J.H.; O'Connell, T.M. (2011). The application of micro-coil NMR probe technology to metabolomics of urine and serum. J Biomol NMR. 49: 297-305.

12. Siderowf, A.; Stern, M.B. (20o6). Preclinical diagnosis of Parkinson's disease: Are we there yet? Curr Neurol Neurosci Rep. 6(4):295-301.

13. Tolosa, E.; Gaig, C.; Santamarıa, J.; Compta, Y. (2009). Diagnosis and the premotor phase of Parkinson disease. Neurology. 72(7 Suppl):S12-S2O.

14. Chaudhuri, K.R.; Healy, D.G.; Schapira, A.H. (2006). National Institute for Clinical Excellence. Non-motor symptoms of Parkinson's disease: diagnosis and management. Lancet Neurol. 5(3):235-245.

15. Monderer, R.; Thorpy, M. (2009). Sleep disorders and daytime sleepiness in Parkinson's disease. Curr Neurol Neurosci Rep. 9(2):173-180.

16. Postuma, R.B.; Gagnon, J.F.; Vendette, M.; Fantini, M.L.; Massicotte-Marquez,J.; Montplaisir, J. (2009). Quantifying the risk of in idiopathic REM sleep behaviour disorder. Neurodegenerative disease Neurology. 72(15):1296-1300.

17. Gagnon, J.F., Vendette, M., Postuma, R.B.; Desjardins, C.; Massicotte-Marquez, J.; Panisset, M.; Montplaisir J. (2009). Mild cognitive impairment in rapid eye movement sleep behaviour disorder and Parkinson's disease. Ann Neurol. 66(1):39-47. 
18. Louis, E.D.; Faust,P.L.; Vonsattel, J.P.; , Rajput, A.; Robinson, C.A.; Rajput, A....\& Hernandez, N. (2007). Neuropathological changes in essential tremor: 33 cases compared with 21 controls. Brain. 130 (12):3297-3307.

19. Benito-Leo, N.J.; Louis, E.D.; Bermejo-Pareja, F. (2009). Neurological Disorders in Central Spain Study Group. Risk of incident Parkinson's disease and Parkinsonism in essential tremor: a population based study. J Neurol Neurosurg Psychiatry. 80(4): 423-425.

20. Ross, G.W.; Petrovitch, H.; Abbott, R.D.; Tanner, C.M.; Popper, J.; Masaki, K....\& White, L.R. (2008). Association of olfactory dysfunction with risk for future Parkinson's disease. Ann Neurol. 63(2):167-173.

21. Haehner, A.; Hummel, T.; Hummel, C.; Sommer, U.; Junghanns. S.; Reichmann, H. (2007). Olfactory loss may be a first sign of idiopathic Parkinson's disease. Mov Disord. 22(6):839-842.

22.Silveira-Moriyama,L.;Petrie, A.; Williams,D.R.; Evans, A.; Katzenschlager, R.; Barbosa, E.R.; Lees, A.J. (2009). The use of a color-coded probability scale to interpret smell tests in suspected Parkinsonism. Mov Disord. 24(8):1144-1153.

23. Goldstein, D.S.; Holmes, C.; Bentho, O.; Sato, T.; Moak, J.; Sharabi, Y....\& Eldadah, B. A. (2008). Biomarkers to detect central dopamine deficiency and distinguish Parkinson disease from multiple system atrophy. Parkinsonism Relate Disord. 14(8):600-607.

24. Mostile, G.; Jankovic, J. (2009). Treatment of dysautonomia associated with Parkinson's disease. Parkinsonism Relat Disord. 15(suppl 3):S224-232.

25. Olanow, C.W.; Prusiner, S.B. (2009). Is Parkinson's disease a prion disorder? Proc Natl Acad Sci U S A. 106(31):12571-12572.

26. Hawkes, C.H.; Del Tredici, K.; Braak, H. (2007). Parkinson's disease: a dual-hit hypothesis. Neuropathol Appl Neurobiol. 33(6):599-614.

27. Michell, A.W.; Luheshi, L.M.; Barker, R.A. (2005). Skin and platelet $\alpha$-synuclein as peripheral biomarkers of Parkinson's disease. Neurosci Lett. 381 (3):294-298.

28. Shishido, T.; Ikemura, M.; Obi, T.; , Yamazaki, K.; Terada, T.; Sugiura, A.....\& Mizoguchi, K. (2010). $\alpha$-Synuclein accumulation in skin nerve fibers revealed by Skin biopsy in pure autonomic failure. Neurology. 74(7):608-610.

29. Marek, K.; Jennings, D.; Tamagnan, G.; Seibyl, J. (2008). Biomarkers for Parkinson’s disease: Tools to assess Parkinson's disease onset and progression. Ann Neurol. 64(Suppl 2):S111-S121.

30. Ibrahim, N.; Kusmirek, J.; Struck, A. F.; Floberg, J. M.; Perlman, S. B.; Gallagher, C.; Hall, L. T. (2016). The sensitivity and specificity of F-DOPA PET in a movement disorder clinic. Am. J. Nucl. Med. Mol. Imaging. 6 (1): 102-109.

31. Arlicot, N.; Vercouillie, J.; Malherbe, C.; Bidault, R.; Gissot, V.; Maia, S.....\& Ribeiro, M.J. (2017). PET imaging of dopamine transporter with 18F-LBT999: first human exploration. $J$. Nucl. Med. 58(1):276.

32. Emamzadeh, F.N.; Surguchov, A. (2018). Parkinson's disease: Biomarkers, Treatment, and Risk Factors. Front. Neurosci. 12:612.

33. Yuncheng, Wu.; Weidong, Le.; Joseph J. (2011). Preclinical biomarkers of Parkinson disease. Archives of neurology. 68(1): 22-30.

34. Marek, K.; Jennings, D. (2009). Can we image premotor Parkinson disease? Neurology. 72(7 Suppl): S21-S26.

35. Schwingenschuh, P.; Ruge, D.; Edwards, M.J.; Terranova, C.; Katschnig, P.; Carrillo, F.; 
....\& Bhatia, K.P. (2010). Distinguishing SWEDDs patients with asymmetric resting tremor from Parkinson's disease: a clinical and electrophysiological study. Mov Disord. 25(5):560569.

36. Skoloudík, D.; Jelínková, M.; Blahuta, J.; Cermák P.; Soukup T.; Bártová P. ...\& Herzig, R. (2014). Transcranial sonography of the substantia nigra: digital image analysis. Am. J.Neuroradiol. 35(12):2273-2278.

37. Chung, E.J.; Kim, E.G.; Bae, J.S.; Eun, C.K.; Lee, K.S....\& Kim, S.J. (2009). Usefulness of diffusion-weighted MRI for differentiation between Parkinson's disease and Parkinson variant of multiple system atrophy. J. Mov. Disord. 2(2):64-68.

38. Saeed, U.; Compagnone, J., Aviv, R. I.; Strafella, A.P.; Black, S.E...\& Masellis, M. (2017). Imaging biomarkers in Parkinson's disease and Parkinsonian syndromes: current and emerging concepts. Transl Neurodegener.; 6:8.

39. Aarsland, D.; Ravina, B. (2010). Biomarkers of PD progression: is CSF the answer Neurology. 75(12): 1036-1037.

40. Li, Q.X.; Mok, S.S.; Laughton, K.M.; Lean, C.A.; Cappai, R.; Masters, C.L.; Culvenor, J.G.; Horne, M.K. (2007). Plasma $\alpha$-synuclein is decreased in subjects with Parkinson's disease. Exp Neurol. 204(2):583-588.

41. Westermann, B.; Wattendorf, E.; Schwerdtfeger, U.; Husner, A.; Fuhr, P.; Gratzl, O.... .\&Welge-Lüssen, A. (2008). Functional imaging of the cerebral olfactory system in patients with Parkinson's disease. J Neurol Neuro-surg Psychiatry. 79(1):19-24.

42. Ozelius, L.J.; Senthil, G.; Saunders-Pullman R.; Ohmann, E,; Deligtisch, A.; Tagliati, M.... ..\&Bressman, S.B. (2006). LRRK2 G2019S as a cause of Parkinson's disease in Ashkenazi Jews. NEngl $J$ Med. 354(4):424-425.

43. Lesage, S.; Dürr, A.; Tazir, M.; Lohmann, E.; Leutenegger, A.L.; Janin, S.; Pollak,P.; Brice, A. (2006). French Parkinson's Disease Genetics Study Group. LRRK2 G2019S as a cause of Parkinson's disease in North African Arabs. N Engl J Med. 354(4):422-423.

44. Veeriah, S.; Taylor, B.S.;Meng, S.;Fang, F.; Yilmaz, E.; Vivanco, I.; ....\& Chan,T.A. (2010) Somatic mutations of the Parkinson's disease-associated gene PARK2 in glioblastoma and other human malignancies. Nat Genet. 42(1):77-82.

45. Sutherland, G.; Mellick, G.; Sue, C.;Chan, D.K; Rowe, D.; Halliday, G. (2007). A functional polymorphism in the parkin gene promoter affects the age of onset of Parkinson's disease. Neurosci Lett. 414(2):170-173.

46. Jankovic, J. (2005). Progression of Parkinson disease: Are we making progress in charting the Course? Arch Neurol. 62(3):351-352.

47. Le, W.; Pan, T.; Huang M.;Xu, P.; Xie, W.; Zhu, W.; Zhang, X.; Deng, H.; Jankovic, J.( 2008) Decreased NURR1 gene expression in patients with Parkinson's disease. J Neurol Sci. 273(1-2):29-33.

48. Scherzer, C.R.; Eklund, A.C.; Morse, L.J.; Liao, Z.; Locascio, J.J.; Fefer, D.; ....\& Gullans, S.R. (2007). Molecular markers of early Parkinson's disease based on gene expression in blood. Proc Natl Acad Sci U S A. 104(3):955-960.

49. Delenclos, M.; Jones, D.R.; McLean, P.J; Uitti R.J. (2016). Biomarkers in Parkinson's disease: Advances and strategies. Parkinsonism Relat Disord. 22 (Suppl 1):S106-110.

50. Hagan, J. J., Leslie, R. A., Patel, S., Evans, M. L., Wattam, T. A., Holmes, S., ....\& Upton, N. (1999). Orexin A activates locus ceruleus cell firing and increases arousal in the rat. Proc. Natl. Acad. Sci. U.S.A. 96(19):10911-10916. 
51. Imperatore, R.; Palomba, L.; Cristino, L. (2017). Role of Orexin-a in hypertension and obesity. Curr. Hypertens. Rep. 19(4):34.

52. Fronczek, R.; Overeem, S.; Lee, S.; Y Hegeman, I.M.; VanPelt, J.; Van Duinen S.G.; Lammers, G.J.; Swaab, D.F. (2007). Hypocretin (orexin) loss in Parkinson's disease. Brain 130, $1577-1585$.

53. Takahashi, Y.; Kanbayashi, T.; Hoshikawa, M.; Imanishi, A.; Sagawa, Y.; Tsutsui, K.; Takeda, Y.; Kusanagi, H.; Nishino, S.; Shimizu, T. (2015). Relationship of orexin (hypocretin) system and astrocyte activation in Parkinson's disease with hypersomnolence. Sleep Biol. Rhythms. 13, 252-260.

54. Shigenaga, M.; Gimeno, C.J.; Ames, B.N. (1989). Urinary8-hydroxy-2'-deoxyguanosine as a biomarker of in vivo oxidative DNA damage. Proc. Natl. Acad. Sci. U.S.A. 86, 9697-9701.

55. Kikuchi, A.; Takeda, A.; Onodera, H.; Kimpara, T.; Hisanaga, K.; Sato, N.; Nunomura, A.; Castellani, R.J.; Perry G.; Smith, M.A.; Itoyama, Y. (2002). Systemic increase of oxidative nucleic acid damage in Parkinson's disease and multiple system atrophy. Neurobiol. Dis 9, 244-248.

56. Nagai, Y.; Ueno, S.; Saeki, Y.; Soga, F.; Hirano, M.; Yanagihara, T. (1996). Decrease of the D3 dopamine receptor mRNA expression in lymphocytes from patients with Parkinson's disease. Neurology. 46, 791-795.

57. Goldstein, D. S.; Holmes, C.; Lopez, G. J.; Wu, T.; Sharabi, Y. (2018). Cerebrospinal fluid biomarkers of central dopamine deficiency predict Parkinson's disease. Parkinsonism Relat. Disord. 50, 108-112.

58. Caronti, B.; Antonini, G.; Calderaro, C.; Ruggieri, S.; Palladini, G.; Pontieri, F.E.; Colosimo, C. (2001). Dopamine transporter immunoreactivity in peripheral blood lymphocytes in Parkinson's disease. Neural. Transm; 108, 803-807.

59. Emamzadeh, F. N. (2017). Role of Apolipoproteins and a-Synuclein in Parkinson's disease. J. Mol. Neurosci. 62, 344-355.

6o. Wang, E.S.; Sun, Y.; Guo, J. G.; GAO, X.; Hu, J.W.; Zhou, L.; Hu, J.; Jiang, C.C. (2010). Tetranectin and apolipoprotein A-I in cerebrospinal fluids potential biomarkers for Parkinson's disease. Acta Neurol.Scand. 122:350-359.

61. Swanson, C. R.; Berlyand, Y.; Xie, S. X.; Alcalay, R. N.; Chahine, L. M.; Chen- Plotkin, A. S. (2015). Plasma apolipoprotein A1 associates with age at onset and motor severity in early Parkinson's disease patients. Mov. Disor. 30, 1648-1656.

62. Dos Santos, M. C. T., Barreto-Sanz, M. A., Correia, B. R. S., Bell, R., Widnall, C., Perez, L. T., ... \& da Costa, A. N. (2018). miRNA-based signatures in cerebrospinal fluid as potential diagnostic tools for early stage Parkinson's disease. Oncotarget, 9(25), 17455.

63. Shadrina, M. I.; Slominsky, P. A. \& Limborska, S. A. (2012). Molecular Mechanisms of Pathogenesis of Parkinson's disease. Int. Rev. Cell Mol. Biol. 281, 229-266.

64. Martins-Branco, D.; Esteves, A. R.; Santos, D.; Arduino, D.M.; Swerdlow, R.H.; Oliveira, C. R. (2012). Ubiquitin proteasome system in Parkinson's disease: a keeper or a witness? Exp. Neurol. 238, 89-99.

65. Blandini, F.; Sinforiani, E.; Pacchetti, C.; Samuele, A.; Bazzini, E.; Zangaglia, R. (2006). Peripheral proteasome and caspase activity in Parkinson disease and Alzheimer disease. Neurology. 66, 529-534.

66. Caudle, W.M.; Bammler, T.K.; Lin, Y.; Pan, S.; Zhang, J. (2010). Using 'omics'to defines pathogenesis and biomarkers of Parkinson's disease. Expert Rev Neurother. 10:925-942. 
67. Goldstein, D.S.; Holmes, C.; Sharabi, Y. (2012). Cerebrospinal fluid biomarkers of central catecholamine deficiency in Parkinson's disease and other synucleinopathies. Brain. 135:1900-1913.

68. Trupp, M.; Jonsson, P.; Ohrfelt, A.; Zetterberg, H.; Obudulu, O.; Malm, L.....\& Forsgren, L. (2014). Metabolite and peptide levels in plasma and CSF differentiating healthy controls from patients with newly diagnosed Parkinson's disease. J Parkinsons Dis. 4:549-560.

69. Öhman, A.; Forsgren, L. (2015). NMR metabolomics of cerebrospinal fluid distinguishes between Parkinson's disease and controls. Neurosci Lett. 594:36-39.

70. Chang, K.H.; Cheng, M.L.; Tang, H.Y.; Huang, C.Y.; Wu, Y.R.; Chen, C.M. (2018). Alternations of metabolic profile and kynurenine metabolism in the plasma of Parkinson's disease. Mol Neurobiol. 55:6319-6328.

71. Oxenkrug, G.; van der Hart, M.; Roeser, J.; Summergrad, P. (2017). Peripheral Tryptophan -Kynurenine Metabolism Associated with Metabolic Syndrome is Different in Parkinson's and Alzheimer's Diseases. Endocrinol Diabetes Metab J. 1(4).

72. Han, W.; Sapkota, S.; Camicioli, R.; Dixon, R.A.; Li, L. (2017). Profiling novel metabolic biomarkers for Parkinson's disease using in-depth metabolomic analysis. Mov Disord.; 32:1720-1728.

73. Cipriani, S.; Chen, X.; Schwarzschild, M.A. (2010). Urate: a novel biomarker of Parkinson's disease risk, diagnosis and prognosis. Biomark Med. 4:701-712.

74. Schwarzschild, M.A.; Schwid, S.R.; Marek, K.; Watts, A.; Lang, A.E.; Oakes, D..... \& Ondrasik, J. (20o8). Serum urate as a predictor of clinical and radiographic progression in Parkinson disease. Arch Neurol.; 65:716-723.

75. Van der Brug, M.P.; Singleton, A.; Gasser, T.; Lewis, P.A. (2015). Parkinson's disease: From human genetics to clinical trials. Sci Transl Med. 7(305): 205.

76. Nagesh Babu, G.; Gupta, M.; Paliwal, V.K.; Singh, S.; Chatterji, T.; Roy, R. (2018). Serum metabolomics study in a group of Parkinson's disease patients from northern India. Clin Chim Acta. 480:214-219.

77. Roede, J.R.; Uppal, K.; Park, Y.; Lee, K.; Tran, V.; Walker, D....\& Jones, D.P. (2013). Serum metabolomics of slow vs rapid motor progression Parkinson's disease: a pilot study. PLoS One. 22; 8(10):e77629.

78. Luan, H.; Liu, L.F.; Tang, Z.; Mok, V.C.; Li, M.; Cai, Z. (2015). Elevated excretion of biopyrrin as a new marker for idiopathic Parkinson's disease. Parkinsonism Relat Disord. 21:13711372 .

79. Luan, H.; Liu, L.F.; Tang, Z.; Zhang, M.; Chua, K.K.; Song, J.X....\& Cai, Z. (2015). Comprehensive urinary metabolomic profiling and identification of potential non-invasive marker for idiopathic Parkinson's disease. Sci Rep. 5:13888.

8o. Unger, M.M.; Spiegel, J.; Dillmann, K.U.; Grundmann, D.; Philippeit, H.; Burmann, J.....\& Schafer, K.H. (2016). Short chain fatty acids and gut microbiota differ between patients with Parkinson's disease and age-matched controls. Parkinsonism Relat Disord. 32:66-72.

81. Hamm-Alvarez., S.F.; Janga, S.R.; Edman, M.C.; Feigenbaum. D.; Freire, D.; Mack, W.J.; Okamoto, C.Y.; Lew, M.F. (2019). Levels of oligomeric $\alpha$-Synuclein in reflex tears distinguish Parkinson's disease patients from healthy controls. Biomark Med. 13(17):1447-1457.

82. Hamm-Alvarez, S.F.; Okamoto, C.T.; Janga, S.R.; Feigenbaum, D.; Edman, M.C.; Freire, D......\& Lew, M.F. (2019). Oligomeric alpha-synuclein is increased in basal tears of Parkinson's patients. Biomark. Med. 13(11): 941- 952. 
83. Al-Nimer, M.S.; Mshatat, S.F.; Abdulla, H.I. (2014). Saliva a-Synuclein and a high extinction coefficient protein. A novel approach in assessment biomarkers of Parkinson's disease. North Am J Med Sci. 6:633-637.

84. Chen, H.; O’Reilly, E.J.; Schwarzschild, M.A.; Ascherio, A. (2008). Peripheral inflammatory bio-markers and risk of Parkinson's disease. Am J Epidemiol. 167(1):90-95.

85. Rentzos, M.; Nikolaou, C.; Andreadou, E.; Paraskevas, G.P.; Rombos, A.; Zoga, M.....\& Vassilopoulos, D. (2009). Circulating interleukin-10 and inter-leukin-12 in Parkinson's disease. Acta Neurol Scand.; 119(5):332-337.

86. Huang, X.; Abbott, R.D.; Petrovitch, H.; Mailman, R.B.; Ross, G.W. (2008). Low LDL cholesterol and increased risk of Parkinson's disease: prospective results from Honolulu-Asia Aging Study. Mov Disord. 23(7):1013-1018.

87. Fang, F.; Zhan, Y.; Hammar, N.; Shen, X.; Wirdefeldt, K.; Walldius, G.; Mariosa, D. (2019). Lipids, Apolipoproteins, and the Risk of Parkinson Disease : A Prospective Cohort Study and a Mendelian Randomization Analysis. Circulation Res. 125: 643-652.

88. Gao, X.; Simon, K.C.; Han, J.; Schwarzschild, M.A.; Ascherio, A. (2009). Genetic determinants of hair color and Parkinson's disease risk. Ann Neurol. 65(1):76-82. 